\title{
A Novel Flux Barrier Parametrization for Synchronous Reluctance Machines
}

\author{
Oğuz Korman, Mauro Di Nardo, Member, IEEE, Michele Degano, Member, IEEE, Chris Gerada, \\ Senior Member, IEEE
}

\begin{abstract}
This paper presents a novel parametrization for the flux barrier profiles of synchronous reluctance and permanent magnet assisted reluctance machines. In literature there are several methods used to design rotor flux barriers of various types, however the vast majority use only a few parameters to characterize their shape. These approaches are proven to be effective in terms of simplicity and computational burden required to achieve an optimal design. However, simplified parametrizations certainly decrease the degrees of freedom when designing the whole barrier shape. In this paper, an attempt to increase the degrees of freedom, introducing a novel rotor flux barrier parametrization, is presented. The method proposed uses natural splines, defined by the positions of a set of control points, to form the shape of the flux barriers. The spline and state-of-the-art barrier profiles are compared from both electromagnetic and mechanical perspectives. The results of this investigation show that by increasing the degrees of freedom it is possible to obtain better performance characteristics. The proposed parametrization is applied to a 6-pole synchronous reluctance motor and its permanent magnet assisted variant, optimized for a traction application. A prototype has been manufactured and tested to experimentally validate the design methodology.
\end{abstract}

Index Terms-Synchronous reluctance, permanent magnet assisted synchronous reluctance, rotor parametrization, flux barriers optimization, spline flux barriers

\section{INTRODUCTION}

Y YNCHRONOUS reluctance (SynRel) machines are one of the $\checkmark$ promising electrical machine topologies with potential for a more efficient and cost effective energy conversion. Thanks to the absence of permanent magnets, SynRel motors present an attractive solution that have found place in both industrial and traction applications.

Since they were first introduced [1], their operating principles and design have been studied in detail by many authors [2-6]. In the last two decades, research on SynRel machines has focused on the rotor design to improve its reluctance features, minimize the torque oscillations and enhance the power factor.

There are mainly three flux barrier profiles used in the literature as they are shown in Fig. 1: circular, straight segmented and barriers shaped based on natural flux lines (will be referred as

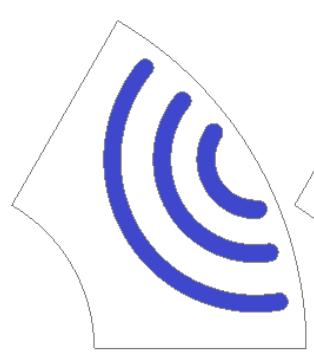

(a)

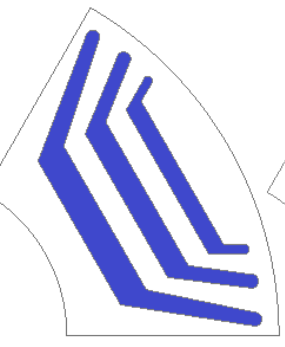

(b)

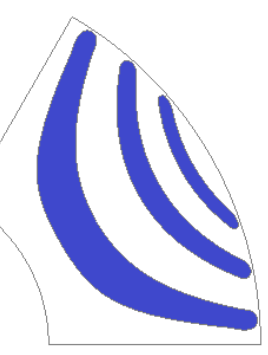

(c)
Fig. 1. Different flux barrier profiles: (a) circular, (b) straight segmented and (c) fluid shape.

fluid).

Circular shaped barriers are one of the most used structures thanks to their simple parametrization. In [6], this barrier type is analytically investigated by using conformal mapping and analytical derivation of the air gap flux density is obtained. Other examples of this barrier type are studied in [7-9] where the effect of barrier's parameters on optimization results are investigated. Flux barriers composed by joining straight segments (also called U-shape) are extensively studied in [10] where the parameters defining the barriers are linked to torque ripple and average torque. One of the most significant features of this barrier type is, they can easily accommodate permanent magnets into the rotor slots transforming SynRel machines into their PM assisted variant. The third flux barrier type is the one parametrized according to Joukowski's flow equations, as reported in [11]. Each of these barrier types have their own advantages and disadvantages in terms of performance and time required to achieve an optimal design. In fact, as discussed in [12] with increased number of parameters it is possible to obtain better designs at the cost of increased computational time.

Another type of barrier profile is described in [13] where second order polynomials are used for shaping the flux barriers. In the study, both symmetrical and asymmetrical pole versions are characterized and analyzed. Alternatively, in [14] hyperbolic flux barriers are modelled analytically by using conformal mapping and magnetic equivalent circuits.

In addition to the barrier types mentioned above, some hybrid configurations have also been presented in the literature. Curved

Oğuz Korman, Mauro Di Nardo, Michele Degano and Chris Gerada are with the Power Electronics Machines and Control Group, Faculty of Engineering, The University of Nottingham, University Park, Nottingham NG7 2RD.

(oguz.korman@nottingham.ac.uk, mauro.dinardo4@nottingham.ac.uk, michele.degano@nottingham.ac.uk, chris.gerada@nottingham.ac.uk) 
and straight flux barriers are adopted in [15] for a permanent magnet assisted SynRel machine claiming the achievement of a better average torque. In [16] asymmetrical configurations are analytically investigated with different barrier shapes mainly for torque ripple reduction. This configuration is further studied in [17] with a sensitivity analysis, showing how the geometric parameters are affecting torque ripple. In another study [18], the decrease in average torque due to an asymmetrical barrier configuration is compensated by using hybrid magnetic core in the stator.

Apart from the studies focusing on different barrier shapes and their effect on performance, analytical methods are being developed to enhance initial sizing of the machine and to decrease the total time required to obtain a fairly good design. An analytical method is developed in [19] dedicated to sizing of SynRel machines, capable of accurately calculating motor performance. In [20], another semi-analytical method is proposed, defining some parameters obtained by minimum FE simulations. Similarly, in [21] a hybrid design procedure bringing analytical and FE method is presented focusing on SynRel machines operating at high speeds.

Although the barrier shapes might differ from each other, there are some specific macro parameters characterizing the shape of a barrier that have major effects on the machine performance. Indeed, regardless of the barrier profile, optimizing the barrier thicknesses increases the saliency and so the torque production capability (for a given rotor diameter) while optimizing the distribution of barriers' end-points on the rotor surface decreases the torque oscillations. These aspects are investigated in [22] and [23] where design rules are analytically derived for determining the thickness of barriers and iron segments along with the barriers' end-positions to achieve an optimal saliency ratio and minimum torque ripple. Effect of these main parameters on the electromagnetic characteristics are investigated by means of sensitivity analysis and optimizations in [8]-[12] and [17] including different flux barrier profiles.

Adopting any of the flux barrier parametrizations presented in the literature and mentioned above, it is not possible to fully control the shape of the whole barrier. Two main philosophy can be adopted to enrich the design possibilities of the SynRel flux barrier profiles. One is to increase the degrees of freedom of a barrier shape which can be parametrized and optimized acting on a given number of variables. The second option is to adopt a topology optimization where the flux barrier profile is not defined by a closed form equation. This work pursues the first option, in fact a richer parametrization is proposed featuring additional degrees of freedom in order to fully investigate the effect of flux barriers' shape on the machine performance. The main idea is to explore if a different rotor core electromagnetic exploitation can lead to improved performance and at what cost.

The proposed parametrization makes use of splines, allowing to define the barrier profile via a set of control points. Using splines to connect these points is advantageous as the resulting shape is always unique, naturally smooth and with high degrees of freedom over the whole barrier. In addition, the introduction of more control points allows to customize the flux barrier shape generation.

A pure SynRel and a permanent magnet assisted synchronous reluctance (PMaSynRel) machine for a medium power traction application are considered to evaluate benefits and drawbacks of adopting this new parametrization. The objective is to investigate the tradeoff between performance, geometric complexity and computational time from electromagnetic and structural point of view. In the first section of the paper, classical and spline parametrizations are explained in detail. Two optimizations are then performed, and results are shown in Section III. The same study is carried out on the PMaSynRel and reported in Section IV. The findings of this work are then experimentally validated on a 6-pole SynRel prototype reported in Section V.

\section{FLUX BARRIERS PARAMETRIZATION}

In this section the most performing state of the art flux barrier parametrization, hereafter called fluid, is presented along with the proposed new variant.

\section{A. Classical Parametrization: Fluid Shaped Barriers}

The derivation of the fluid barriers' profile is based on Joukowski's flow equation first reported in [11]. Indeed, the flux lines in a solid rotor when supplying a distributed three phase winding placed in the stator can be expressed via (1) and (2).

$$
\begin{array}{r}
C=\frac{\sin (p \theta)\left[\left(\frac{r}{R_{\text {shaft }}}\right)^{2 p}-1\right]}{\left(\frac{r}{R_{\text {shaft }}}\right)^{p}} \\
r(\theta)=R_{\text {shaft }} \sqrt[p]{\frac{C+\sqrt{C^{2}+4 \sin ^{2}(p \theta)}}{2 \sin (p \theta)}}
\end{array}
$$

Equation (1) is used to determine the parameter $\mathrm{C}$, which is a constant related to a flux line passing through any polar coordinate $(r, \theta)$, where $p$ is the number of pole pairs and $R_{\text {shaft }}$ is the shaft radius. If the parameters $(r, \theta)$ are selected on the rotor surface then $\mathrm{C}$ can be easily calculated and then (2) can be used to find all points defining the flux line specified by the constant $\mathrm{C}$ using $\theta$ in the range $[0, \pi / \mathrm{p}]$.

Each barrier can be therefore defined by two parameters: endpoint angle (will be referred as EPA from now on) and thickness of the barriers in per unit of the maximum available space. In particular, the following procedure is here adopted to obtain a three flux barrier rotor but can be extended to any number of barriers:

1) Once the EPA for the outermost barrier is selected, the other barriers' EPAs are calculated with (3) where a coefficient (EPA_C) is used in per units to represent them. EPA_C for remaining barriers can have a maximum value of $1 /$ (\#barriers1) in per units, where the minimum value should be greater than 0. By doing so, the EPA of the innermost barrier is 
constrained to a desired maximum value. Fig. 2 shows the EPA with red dots and the corresponding flux profiles with dashed lines.

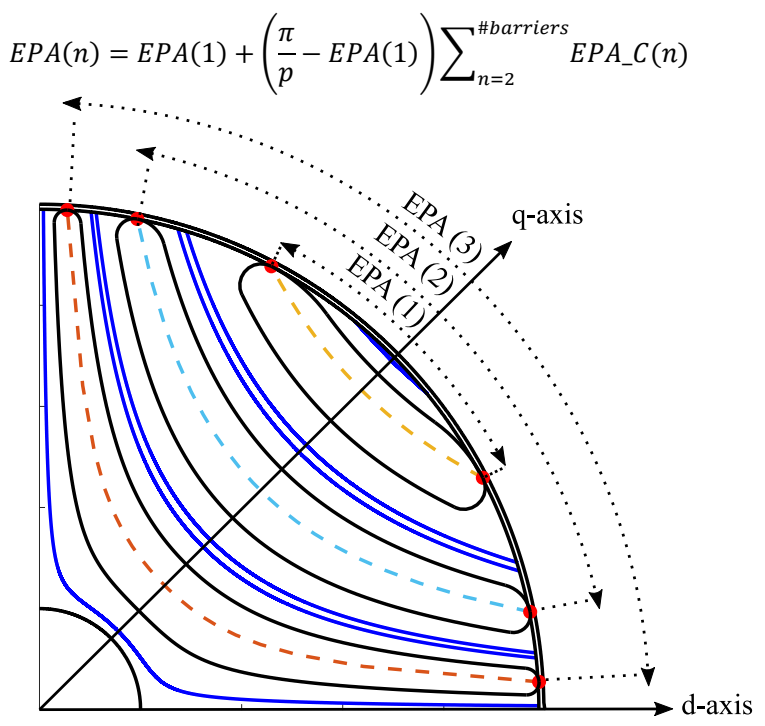

Fig. 2. SynRel machine sketch: rotor flux barriers parametrization with fluid shape.

2) Maximum thickness available (will be referred as MTA) for the upper and lower part of the barrier can be calculated by finding the middle point of the field line at $(\mathrm{r}, \theta=\pi / 2 p)$ by using (2) then by using (4) where $n=2$ to (\#barriers-1). MTA for the outermost barrier's upper side and the innermost barrier's lower side can be similarly calculated by using rotor radius and shaft radius. Additional safety terms might be used to further prevent any overlap and guarantee a minimum iron thickness. Flux lines passing through the maximum thicknesses are shown with blue lines in Fig. 2.

$$
\operatorname{MTA}(n)=\frac{\left(r(n), \theta=\frac{\pi}{2 p}\right)-\left(r(n+1), \theta=\frac{\pi}{2 p}\right)}{2}
$$

3) Once the MTA is found for all barriers, thickness coefficients can be used in per unit system in the range of $(0,1]$ to parametrize the thickness of each barrier. Thickness coefficient being equal to 1 corresponds to maximum thickness that a barrier can have. Lower and upper flux lines forming the barriers are shown with black lines in Fig. 2.

4) For the end-shape of the barriers: two arcs are calculated and joined being tangent to the neighboring flux line and end-point. This way end-shapes are always made to be round as seen in Fig. 2.

The parametrization of fluid shape barriers consisting of three barriers are defined and explained with 6 parameters. The number of parameters defining the barrier's shape can be decreased or increased by slightly modifying this basic parameterization. For example, it can be increased to 9 by defining an additional shifting parameter making the middle line of the barrier not centered respect to its upper and lower side as mentioned in [8] and [12] or can be decreased to 4 by using only one thickness parameter for controlling thicknesses of all barriers.

\section{B. Spline Parametrization}

Natural cubic spline is a set of piecewise third-order polynomials curve defined by the position of control points. It is selected due to its smooth resultant curve always passing through the defined control points which makes it easy to control the barrier shape within a defined space. The proposed spline parametrization is built by defining the position of $m$ control points for each barrier. To do so, first a classical parametrization has to be defined so that it can be used as a base. The following steps are describing in detail the spline parametrization for a single barrier:

1) The classical parametrization is described by 2 parameters per barrier, EPA and thickness. These parameters will also be used to describe the new parametrization. In Fig. 3 (a), flux barrier profiles are shown where black lines are the actual barrier lines, blue lines are the thickness limits and the red line is the middle line.

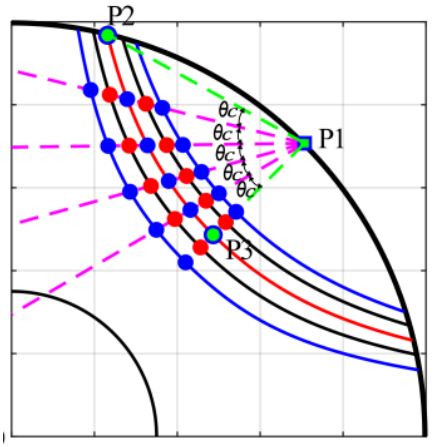

(a)

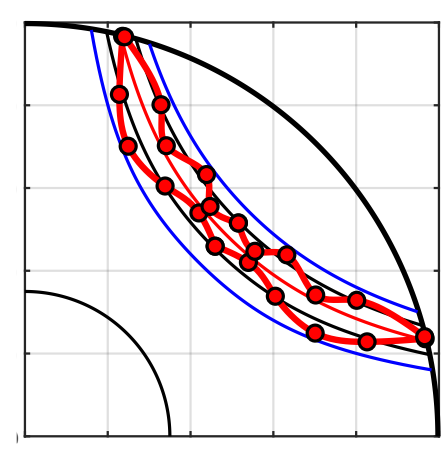

(b)
Fig. 3. Steps by step description to form the spline parametrization proposed.

2) In order to maintain convex nature of the flux barriers, the position of the control points is determined by the intersection of straight lines drawn from $P_{1}$ (middle point of the rotor surface) and the flux lines. Straight lines are generated to intersect red flux line, therefore the end-point of the line and the point in the middle are two extreme points. For each straight line, two control points are identified, on lower and upper black colored flux lines which are representing the flux lines of a classical fluid shape barrier profile. This means that for $m$ number of control points, where $m$ is always an even number, there are $m / 2$ pairs of control points that lay on the same straight line drawn from $P_{1}$. These control points are shown with red dots in Fig. 3 (b). In order to form the spline barrier profile, these control points (red dots) have the flexibility to move between adjacent blue dots that are on the blue flux lines. The latter represent each barrier's maximum limits for upper and lower side of the barrier.

3) $\angle P_{2} P_{1} P_{3}$ is known since it is related to the EPA and middle point angle of the red flux line and it can be represented as $90^{\circ}-\frac{E P A}{4}$ in degrees. To form $m$ control points, $m / 2$ number 
of straight lines have to be defined starting from $P_{1}$ making an angle of $\theta_{c}=\frac{\angle P_{2} P_{1} P_{3}}{m / 2}=\frac{90-\frac{E P A}{4}}{m / 2}$ with each other. Straight lines can be expressed in the form of (5) with the gradient (6) and constant (7) where $i=1 \ldots \frac{m}{2}$ is the integer index of the straight lines. For the constant $c_{i}, P_{1(x)}$ and $P_{1(y)}$ are $\mathrm{x}$ and $\mathrm{y}$ coordinates of the $P_{1}$ respectively.

$$
\begin{gathered}
y_{i}(x)=m_{i} * x+c_{i} \\
m_{i}=\tan \left(\frac{180}{p}-\theta_{c} *(i-1)\right) \\
c_{i}=P_{1(y)}-m_{i} * P_{1(x)}
\end{gathered}
$$

4) As described in section-II A, flux lines are represented by a constant $\mathrm{C}$ by using (1) and (2). In order to determine the intersection of a flux line and a straight line (8) has to be solved. However, there is no analytical solution to this equation, because a flux line defined by $\mathrm{C}$ exhibits trigonometric and exponential behaviour between its $\mathrm{x}$ and $\mathrm{y}$ coordinates. For this reason (8) is solved numerically.

$$
\mathrm{C}(\mathrm{x}, \mathrm{y})=m_{i} * x+c_{i}
$$

5) After the position of control points are identified, next step is to generate piecewise cubic splines. For $m$ number of control points there are $\left(\frac{m}{2}+1\right)$ points for upper and lower part of the flux barrier, where the additional term stands for the end-point. By using these points, two sets of piecewise cubic splines are realized for upper and lower parts of flux barrier and the joined together. Each set of splines have $\left(\frac{m}{2}\right)$ number of piecewise cubic polynomials. Curve fitting of the splines is done by using the spline function of MATLAB.

6) As discussed earlier, control points (red dots) have the freedom to move between adjacent blue dots creating a straight path on the lines characterized by (5). The movement of the control points is parametrized based on the path they can move. Each control point can have a value in the range [-1 1]. Three important positions of the control points are when they take a value $-1,1$ and 0 . If the control point takes the value 0 , then it will not move and stay at the original position it is defined. When it takes a value of -1 and 1 , it becomes exactly on the lower and upper blue dots respectively. In general, control points move towards lower and upper blue dots based on its negative or positive value.

As an example, ten control points are defined by the intersection of five straight lines and flux lines and are shown in Fig. 3. Value of the control points are randomized in the range [-1 1] for demonstration purposes.

With the intention of discussing the impact of different number of control points, various spline barrier profiles are generated with 6, 10, 20 and 30 control points per barrier. This is shown in Fig. 4, through (a) and (b) with increasing number of control points. Fluid shape barriers are also shown for comparison. The values of the control points are randomly generated, and EPAs of the same order barriers are same. It is important to notice as the number of control points increases as the complexity of the barrier profiles increase. Another point of consideration is that, increased number of control points does not have the same effect on each barrier's profile. Therefore, it would be beneficial in terms of controlling the complexity of the barrier shapes, to select the number of control points separately for each barrier.

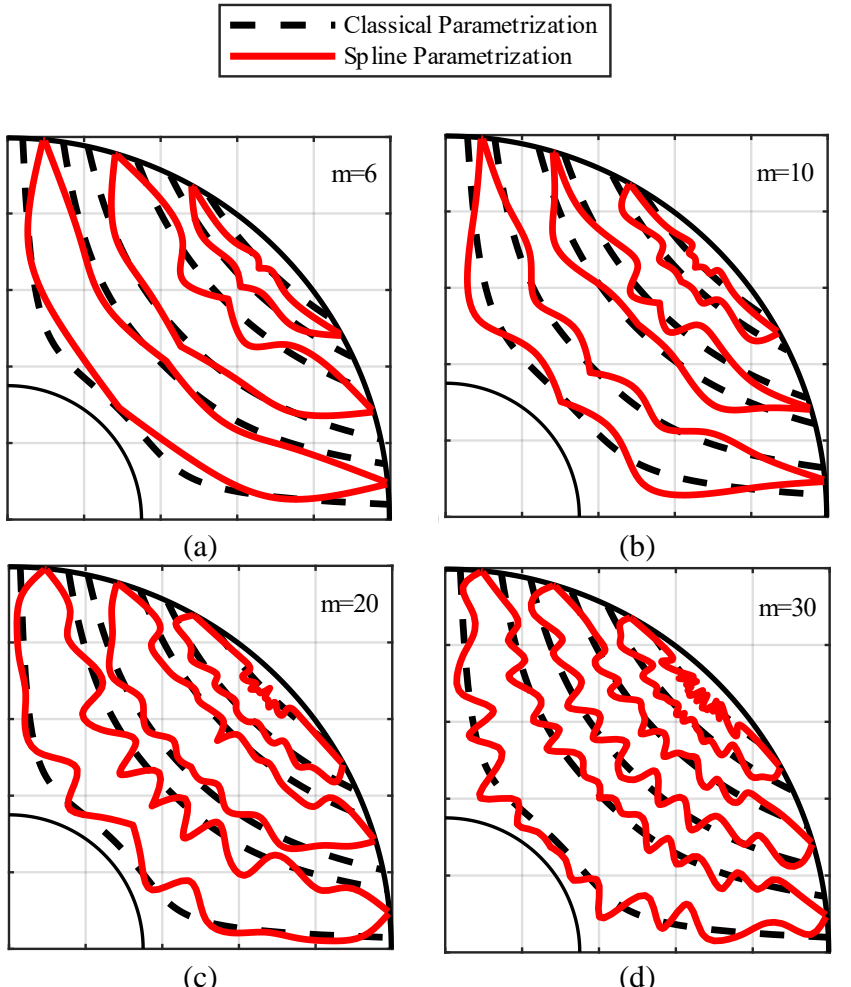

Fig. 4. Spline barrier profiles examples formed with different control points number per barrier: (a) $m=6$, (b) $m=10$, (c) $m=20$, (d) $m=30$

\section{MACHINE SPECIFICATIONS AND OPTIMIZATION SETtINGS}

A summary of the machine specifications considered in this case study are based on requirements for light traction applications as reported in TABLE 1 . The overall housing and stator dimensions of the machine are based on an existing permanent magnet motor designed to fulfil the application requirements. A maximum current density of $10 \mathrm{~A} / \mathrm{mm}^{2}$ is considered with a distributed, single layer winding. Cooling is provided by a water jacket with a flow rate of $3 \mathrm{~L} / \mathrm{min}$. Both stator and rotor laminations are using an M290-50A steel. The stator dimensions are kept constant throughout the optimizations, where only the rotor is modified. The optimizations focus on the design of the flux barrier profiles and their effect on torque production capability and torque ripple.

Optimization of electric machines generally involves more than one objective function, most of the time in conflict with each other in a non-linear behavior. Therefore, there is not a unique solution to the problem but rather a set of Pareto-optimal solutions.

For this reason, a multi-objective stochastic optimization algorithm (MOGA) has been used and embedded in a commercial 
suit (ModeFrontier) [24]. Adopting this approach, it is possible to determine a set of non-dominated design solutions without making any preliminary assumption on the relative importance of the considered objectives.

The optimization parameters are summarized in TABLE II:

- For the optimization considering fluid shape flux barrier profiles, 6 parameters are used: EPA_C and thickness for three barriers. EPA_C for the outermost barrier is limited to have a range of [0.3 0.8] and EPA_C for the other barriers are limited in the range [ 0.10 .5$]$ whereas thicknesses of each barrier is in the limits [0.1 0.9$]$.

- For the spline flux barrier profiles: 4, 6 and 8 control points are used for outermost, middle and innermost barriers respectively, where they can take a value in the range [-0.9 0.9]. In addition, the EPA_C and thicknesses for three barriers are also optimization variables making a total of 24 parameters. The number of control points is selected such that the shape of the proposed barrier profile does not get over complicated to cause any problems in the mesh or lead to local optima during the optimization given the high research space dimension.

- Both optimizations have the same target functions: maximization of average torque and minimization of torque ripple. For the maximization of average torque, a minimum of $98 \mathrm{Nm}$ has been imposed as a constraint in order to determine machine designs with higher torque values. Similarly, a maximum torque ripple constraint of $20 \%$ has been considered.

The total number of designs to be evaluated is determined according to the number of optimization variables. For the optimization of classical fluid shape barrier profile, a total of 3600 designs are to be evaluated through 60 generations and 60 individuals. Since there are higher number of variables for the proposed spline barrier profile a higher number of total designs are considered. 21875 total designs are selected to be evaluated through 125 generations each having 175 individuals. Initial population for both parametrizations is generated via Sobol Sequences [25] in order to uniformly distributed optimization variables.

A 2D FEA analysis is done for each design, considering one sixth of the motor to make use of periodicity and $1^{\circ}$ mechanical angle steps in order to properly capture the torque harmonics.

Another important point of consideration is the selection of current phase angle related to the maximum torque for a given current (MTPA). This can be evaluated by doing multiple FEanalysis for each design within a range of current phase angles. Alternatively, the current phase angle can be included within the optimization variable to be identified by the algorithm as reported in [7] and [9]. The former method would clearly increase the computation time while the latter increases the number of optimization variables. In the presented study, the current phase angle is kept constant at $60^{\circ}$ electrical degrees during the optimization. For different rotor geometries the MTPA phase angle will vary between $56^{\circ}$ and $63^{\circ}$ electrical degrees, and as a consequence also the average torque can change. However, it has been verified that machines with lower torque ripple and highest average torque have an MTPA phase angle in a range of $\pm 1^{\circ}$ electrical degree.
TABLE I. DESIGN SPECIFICATIONS

\begin{tabular}{|c|c|c|}
\hline Parameter & Value & Unit \\
\hline Rated Speed [RPM] & 2500 & $\mathrm{rpm}$ \\
\hline Maximum Speed [RPM] & 10000 & $\mathrm{rpm}$ \\
\hline DC Bus Voltage [V] & 610 & $\mathrm{~V}$ \\
\hline Stator diameter [mm] & 245 & $\mathrm{~mm}$ \\
\hline Rotor diameter [mm] & 160 & $\mathrm{~mm}$ \\
\hline Air gap length [mm] & 0.7 & $\mathrm{~mm}$ \\
\hline Stack kength [mm] & 120 & $\mathrm{~mm}$ \\
\hline Number of slots & 36 & - \\
\hline Number of poles & 6 & - \\
\hline
\end{tabular}

TABLE II. OPTIMIZATION PARAMETERS

\begin{tabular}{|c|c|c|}
\hline Parameter & $\begin{array}{c}\text { Classical Fluid Shape } \\
\text { Parametrization }\end{array}$ & Spline Parametrization \\
\hline $\begin{array}{l}\text { Number of } \\
\text { Parameters }\end{array}$ & ( & $\begin{array}{c}24(6+18) \\
4,6 \text { and } 8 \text { control points } \\
\text { from outermost barrier to } \\
\text { inner barrier }\end{array}$ \\
\hline $\begin{array}{l}\text { Objective } \\
\text { Functions }\end{array}$ & $\begin{array}{ll}-\quad & \begin{array}{l}\text { Maximisation of } \\
\text { average torque }\end{array} \\
\text { - } & \begin{array}{l}\text { Minimisation of } \\
\text { torque ripple }\end{array}\end{array}$ & $\begin{array}{l}-\quad \begin{array}{l}\text { Maximisation of } \\
\text { average torque }\end{array} \\
\text { Minimisation of } \\
\text { torque ripple }\end{array}$ \\
\hline $\begin{array}{l}\text { Objective } \\
\text { Constraints }\end{array}$ & $\begin{array}{ll}- & \text { torque ripple }<20 \% . \\
- & \text { average torque }>98 \\
\mathrm{Nm}\end{array}$ & $\begin{array}{ll}- & \text { torque ripple }<20 \% \\
- & \text { average torque }>98 \\
\mathrm{Nm}\end{array}$ \\
\hline $\begin{array}{c}\text { EPA_C } \\
\text { (End-Point Angle } \\
\text { Coefficient) } \\
\end{array}$ & $\begin{array}{ll}- & \text { EPA_C }(1)\left[\begin{array}{ll}0.3 & 0.8\end{array}\right] \\
- & \text { EPA_C }(2-3)\left[\begin{array}{ll}0.1 & 0.5\end{array}\right]\end{array}$ & $\begin{array}{ll}- & \text { EPA_C(1) }\left[\begin{array}{ll}0.3 & 0.8\end{array}\right] \\
- & \text { EPA_C(2-3) }\left[\begin{array}{ll}0.1 & 0.5\end{array}\right]\end{array}$ \\
\hline Thickness & {$\left[\begin{array}{ll}0.1 & 0.9\end{array}\right]$} & {$\left[\begin{array}{ll}0.1 & 0.9\end{array}\right]$} \\
\hline Control Points & - & {$\left[\begin{array}{lll}-0.9 & 0.9\end{array}\right]$} \\
\hline
\end{tabular}

\section{SYNREL ROTOR OPTIMIZATION AND FURTHER ANALYSIS}

\section{A. Optimization Results}

The results of the optimizations are shown in Fig. 5, where the Pareto fronts are reported. Torque ripple and average torque, which are the main objective functions are represented for both optimizations. It is seen that the evaluated designs are comparable to each other in terms of average torque and torque ripple. It can be inferred that the spline parametrization provides higher average torque for medium high torque ripple design. On the other hand, classical fluid shape geometry can offer better designs in terms of torque ripple.

Two designs are selected from each Pareto front as marked in Fig. 5 (star marked). They have almost the same torque ripple with $9.8 \%$ but different average torques, $100.5 \mathrm{Nm}$ for the classical and 101.2 $\mathrm{Nm}$ for the spline parametrization, respectively. The shapes of the two geometries are shown in Fig. 6. It can be noticed that the barriers formed with splines have more air compared to classical fluid shaped barriers. This is the main reason that spline parametrization offers better average torque as it has the capability to increase the saliency ratio, enhancing the reluctance along the 
q-axis, but without obstructing the d-axis iron path, as it would happen with the classical parametrization.

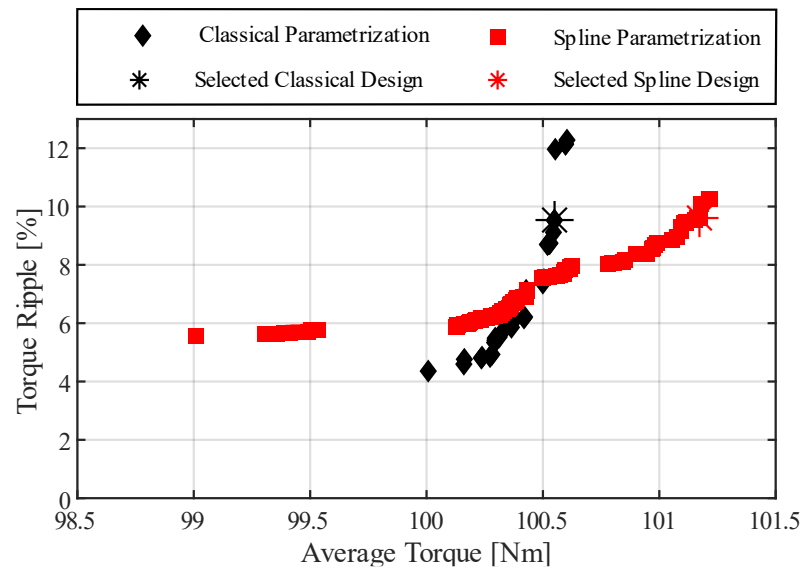

Fig. 5. Pareto fronts of the classical and spline parametrization.

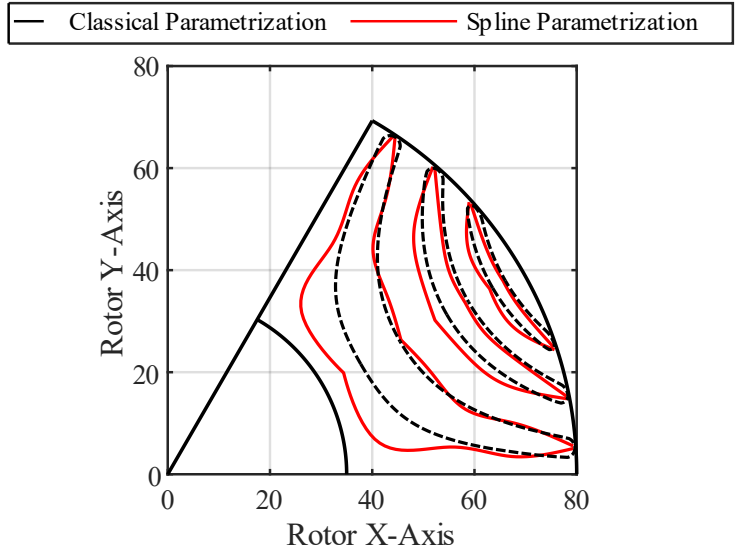

Fig. 6. Pareto front designs of classical fluid shape and spline parametrization

The end-points for both parametrizations are located in the same region of the rotor periphery. Even if the end-barriers are slightly different, these sets of end-points give the same torque ripple. This is reasonable and justified by the fact that both fluid and sharp endprofiles are saturating, as described in detail in [26].

\section{B. Analysis of the Optimal Machines}

The selected designs are further analyzed in terms of electromagnetic and mechanical performance. Electromagnetic analysis (2D FEA) is undertaken with a finer mesh over two electrical periods with a mechanical step of $1^{\circ}$. Amplitude of the peak current is varied between $10 \mathrm{~A}$ and $160 \mathrm{~A}$ with $10 \mathrm{~A}$ steps. In order to evaluate the MTPA condition, current phase angle is analyzed in the range $45^{\circ}$ and $75^{\circ}$, divided to 30 equally spaced points for each current amplitude. After that, MTPA point for each current amplitude is calculated. Average of iron losses are evaluated over the second electrical period of the analysis. Torque versus mechanical position for both designs at nominal condition $\left(82 \mathrm{~A}_{\text {peak }}\right)$ are shown in Fig. 7 . At this condition current phase angle for MTPA operation is found to be $57.4^{\circ}$ for both designs. Average torque is $101.1 \mathrm{Nm}$ and $101.6 \mathrm{Nm}$, and torque ripple is $10.5 \%$ and $10.8 \%$ for classical and spline designs, respectively.

Regarding the magnetic characteristics of the selected designs, average torque, torque ripple, flux linkages, inductances, iron losses and power factor are shown in Fig. 8 at MTPA conditions. Fig. 8 (a) shows the average torque versus increasing peak current where difference between two designs is also included. Average torque for spline parametrization is around $0.4 \%$ higher than classical parametrization at around nominal conditions, however due to different saturation characteristics of the machines this value increases towards higher currents. A similar effect is also spotted for torque ripple as shown in Fig. 8 (b). Although the torque ripples are very close at nominal conditions, classical design exhibits higher torque ripple with increasing current. Flux linkages and inductances are presented in Fig. 8 (c) and (d). It is seen that both d-axis and q-axis flux linkages and inductances of the classical design are higher than spline design, however difference of inductances $\left(\mathrm{L}_{\mathrm{d}}-\mathrm{L}_{\mathrm{q}}\right)$ for spline design is higher than that of classical design, resulting in higher average torque. Another point of consideration is the iron losses which are shown in Fig. 8 (e). Except low current amplitudes, classical design, starting from $60 \mathrm{~A}_{\text {peak }}$ have higher iron losses than spline design. An additional noticeable difference of the two designs is seen in Fig. 8 (f) where spline design has higher power factor than classical design at every current amplitude.

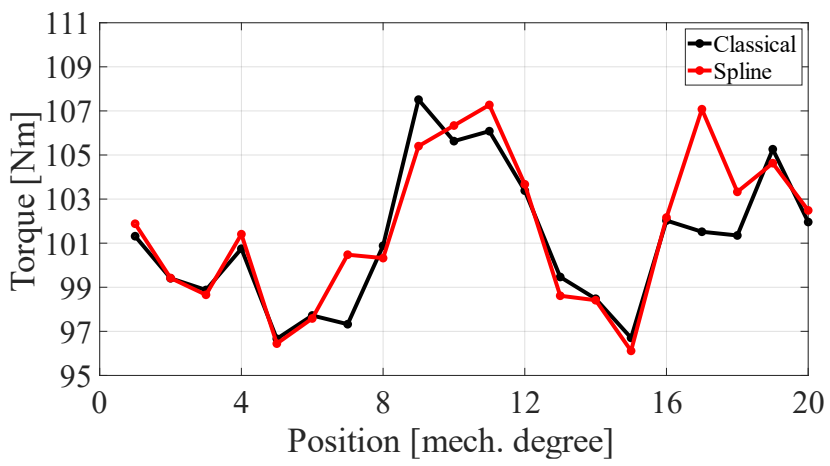

Fig. 7. Torque versus position for selected classical and spline machines.

The magnetic field distribution of the designs is shown in Fig. 9. It is seen that there are some local differences in the saturation levels especially in the end-point parts of the barriers. Classical and spline design have a maximum flux density of $2.4 \mathrm{~T}$ and $2.6 \mathrm{~T}$ respectively in the ribs region as shown with black circles in Fig. 9 (a) and (b). Average flux densities of classical and spline design are $0.97 \mathrm{~T}$ and $1.21 \mathrm{~T}$ for the rotor, whereas average flux density of the stator is $1.07 \mathrm{~T}$ and $1.11 \mathrm{~T}$, respectively. This is mainly caused by different end-shapes of two barrier profiles as well as the different iron thicknesses between the flux barriers. This is mostly evident in the iron part between shaft and innermost barrier, where the classical fluid profile has lower flux density. The spline profile instead allows to make the best use of the iron leading to a more distributed saturation in that region.

To validate mechanical robustness of the rotor, 2D structural analysis have been carried out by means of FEA at the maximum speed of $10000 \mathrm{rpm}$. A set of iron bridges have been added to the rotor structure and their thicknesses have been preliminary 
identified for each flux barrier based on the mass of each rotor island as described in [27]. Considered rib distribution is shown in Fig. 10, where the bridges have $2 \mathrm{~mm}, 1 \mathrm{~mm}$ and $1 \mathrm{~mm}$ thicknesses for innermost, middle and outermost barriers, respectively. Considering the $465 \mathrm{MPa}$ yield stress of laminated steel, classical geometry satisfies this limit with 1.35 safety factor whereas spline geometry cannot with a safety factor of 0.75 . The difference between the maximum stress levels is located at the end-parts of the barrier profiles. Both designs have a tangential rib thickness of $0.5 \mathrm{~mm}$, however due to the end-shapes of two parametrizations, stress values critically change, confirming the results discussed in [26].

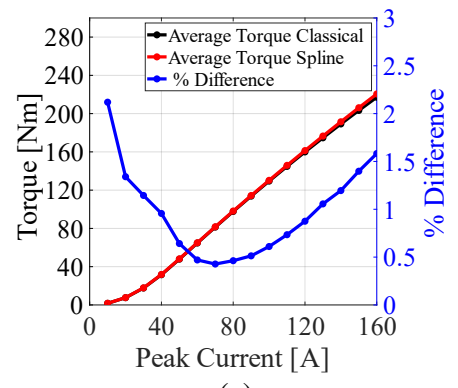

(a)

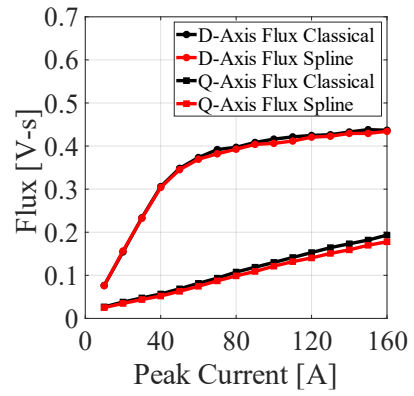

(c)

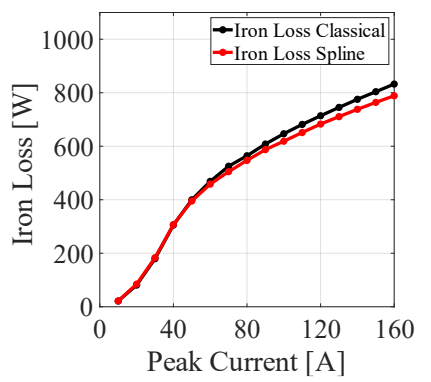

(e)

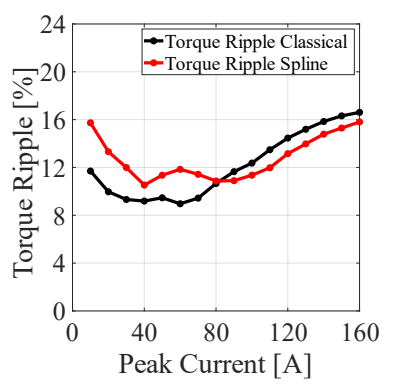

(b)

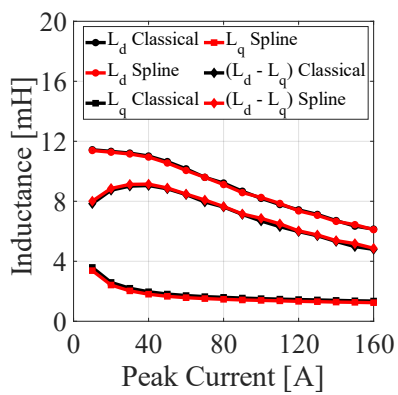

(d)

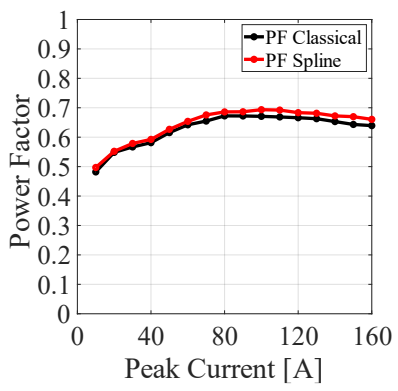

(f)
Fig. 8. Performance characteristics of two selected designs for classical and spline parametrizations: (a) Average torque, (b) Torque ripple, (c) D-Q axis flux linkages, (d) D-Q axis inductances, (e) Iron loss and (f) Power factor

In the end-region of the fluid barrier profile, forces acting to the end-parts are uniformly distributed. In contrast, the sharp end of the spline profile design causes the force distribution to act on a smaller area causing the stress to be higher than fluid profile design. When other critical parts are examined, such as the radial iron bridges, the stress distributions are mainly the same. This has particular importance because although there is less iron in spline profile design, similar stress levels are obtained other than the endregions.

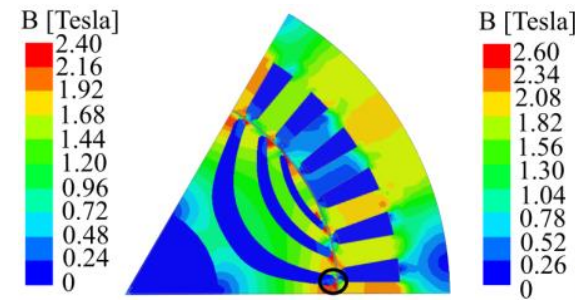

(a)

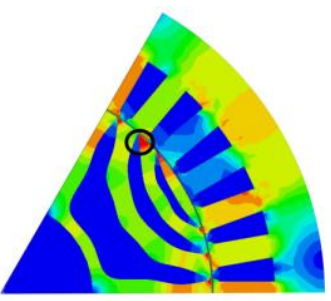

(b)
Fig. 9. Magnetic field distributions of selected classical and spline machines.

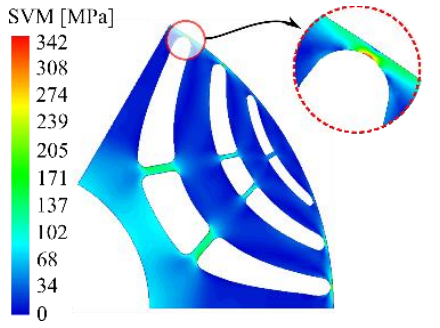

(a)

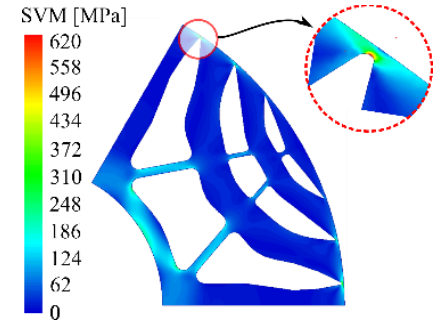

(b)
Fig. 10. Von Mises stress distribution results: (a) classical parametrization, (b) spline parametrization

\section{PMASYNREL ROTOR OPTIMIZATION}

This section explains the procedure for building the flux guides of a PMaSynRel machine with splines. Rectangular magnets are considered with flux guides having both classical fluid profiles and spline profiles.

\section{A. Parametrizations for PMaSynRel}

The details of classical and spline flux barrier profiles are explained in Section II. In case the design requires PMs to improve performance with respect to the SynRel version, some minor changes are required.

Apart from the inclusion of rotor slots where to insert the PMs, the flux guides with fluid profile can be built by following the same procedure as explained in Section II. Once the flux barrier profile is known by using EPAs and thicknesses, rectangular magnet dimensions can be identified. Thickness of the magnet is the thickness of the barrier and length of the magnet is determined by introducing a coefficient as a ratio between the magnet length and fluid line length. After the magnet is drawn depending on the maximum speed of the application the iron bridges can be computed considering the center of mass of each rotor island as in [27]. Later, fluid lines are redrawn from the end of the bridge towards the end-points.

As for the flux guides with spline profiles, first fluid shape flux guides with magnets are formed. After that, as described in Section II, control points are added, and the spline guide profile is formed. In Fig. 11 two example rotor structures embedding rectangular rotor slots to host the PMs are shown. Fig. 11 b shows how the 
spline guide profiles are formed with 4 control points per barrier as highlighted with blue dots.

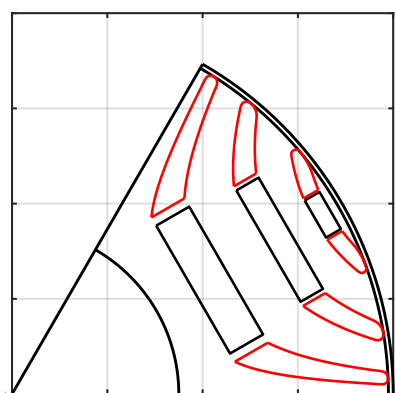

(a)

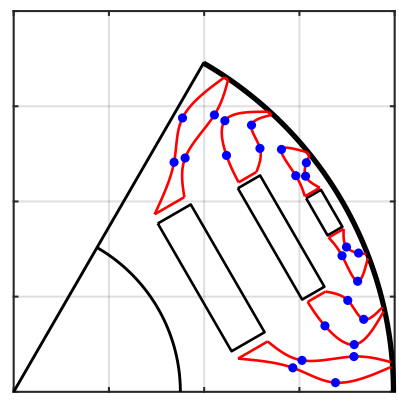

(b)
Fig. 11. Sample rotors obtained: (a) classical parametrization (b) spline parametrization.

\section{B. PMaSynRel Motors Optimization}

Two optimizations utilizing fluid and spline guides are carried out to analyse and understand the effect of modifying the barrier shapes in case of PMaSynRel motors. Specifications of the machine are the same as that given in Section-III. A low-grade ferrite magnets (Y30 grade) are used. Three additional parameters accounting for magnet length are added to each parametrization having a per unit range of [0.2 0.6]. For the spline parametrization 2, 3 and 4 control points are used respectively for the outermost, middle and innermost barriers. Less control points are used when compared to the previous optimizations as the spline profiles are distributed over a smaller area.

With the modifications made, 9 variables are used for optimizing the classical fluid parametrization and 18 variables are used for spline parametrization. Consequently, 9000 and 18000 designs are evaluated for two optimizations. As the optimization is focusing on the effect of rotor design on torque characteristics, two target functions are considered aiming to improve average torque and reduce torque ripple.

Optimization results are presented in Fig. 12, showing the average torque and corresponding torque ripple values of two optimizations. Parametrization governing spline profile flux guides has achieved better average torque (4\%) and slightly better torque ripple when compared to the parametrization with fluid shaped flux guides.

One of the designs, on the Pareto front of spline parametrization is selected for further analysis as shown in Fig. 13. Selected machine is analyzed with and without magnets and resulting waveforms are reported in Fig. 14. Average torque of the machine with ferrite magnets is higher as expected due to the increased qaxis flux. However due to the change in the saturation levels in some critical parts which dominantly affect torque ripple such as ribs and bridges, machine with no magnets exhibit higher torque ripple.

\section{EXPERIMENTAL VALIDATION}

For verification of the analysis results, selected design shown in Fig. 13 is manufactured and tested without magnets. A picture of the rotor manufactured, before inserted into the stator and housing is shown in Fig. 15, where the flux barriers are clearly identified.
In order to further decrease the torque ripple in the machine, a discrete skewing of the rotor is evaluated with a set of parametric $\mathrm{FE}$ analysis and the final machine is built with $4^{\circ}$ skew angle in 3 step pieces.

A picture of the test rig used to characterize the prototype is shown in Fig. 16. The magnetic characteristics are identified in the first quadrant of the d-q current plane, using the test methodology described in [28].

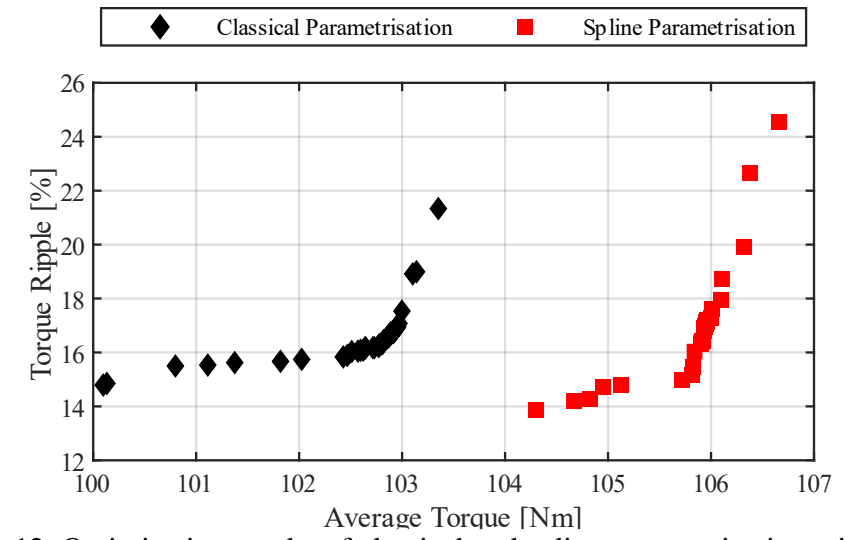

Fig. 12. Optimization results of classical and spline parametrization with magnets.

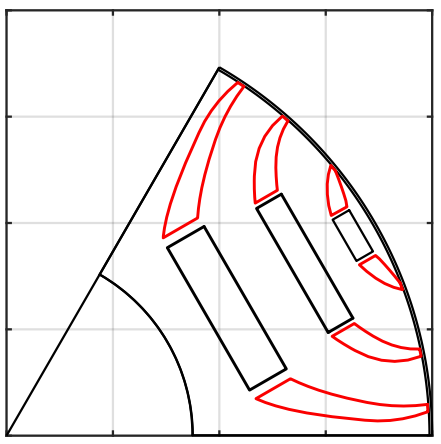

Fig. 13. Selected design obtained by spline parametrization.

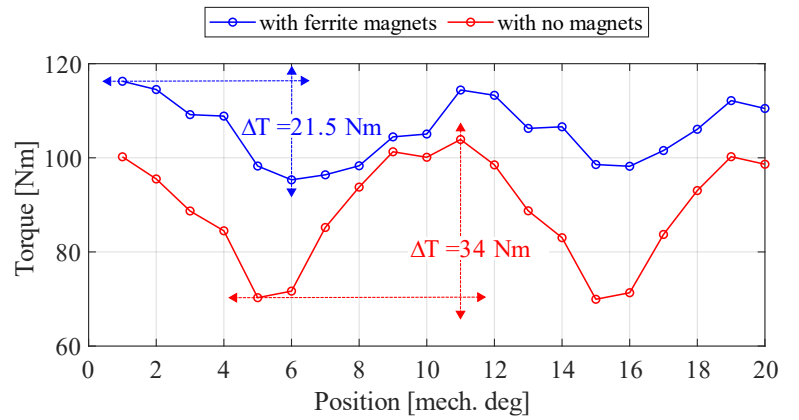

Fig. 14. Torque versus position for different cases.

The prototype speed is set by a load motor in speed control mode at $1000 \mathrm{rpm}$. Sets of Id, Iq currents, with steps of 8A, are set through the converter controlling the prototype up to a maximum current vector of 82Apeak. During the measurements, the torque at the shaft is measured and acquired by the torquemeter interface. 


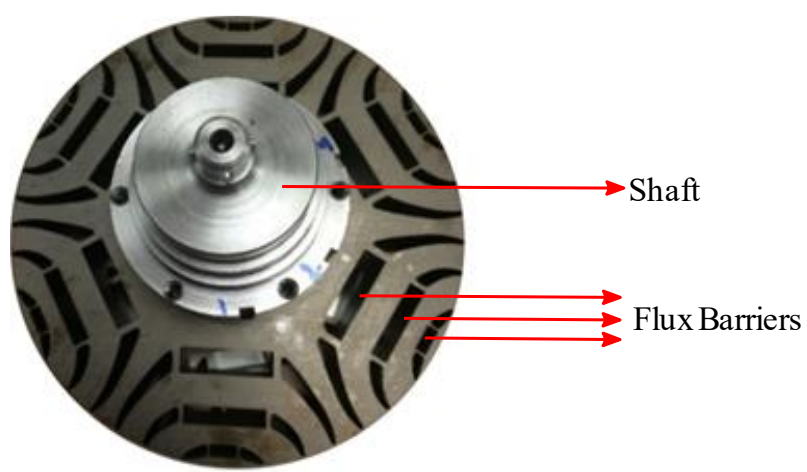

Fig. 15. Prototype of the selected design.

Experimental measurements are shown in Fig. 17 with the corresponding FEA results. In the Fig. 17 (a), motor characteristics are shown in the first quadrant of the plane. Constant torque curves are shown until $100 \mathrm{Nm}$, where the machine produces about $90 \mathrm{Nm}$ when the supplied current is $82 \mathrm{~A}_{\text {peak }}$.

It is seen that, especially towards higher current levels the prototyped machine provides less torque than expected. This discrepancy is less than $5 \%$ when considering the same current amplitude. Torque characteristics as function of the current phase angle is shown in Fig. 17 (b). Here, both FEA and experimental tests curves show very similar characteristics. At about $60^{\circ}$ (closer to the q-axis), both machines operate at MTPA point providing an output torque being the maximum torque point. Consequently, $88.5 \mathrm{Nm}$ and $84.5 \mathrm{Nm}$ average torques (4.5\% difference) values are obtained from FEA and test results, respectively.

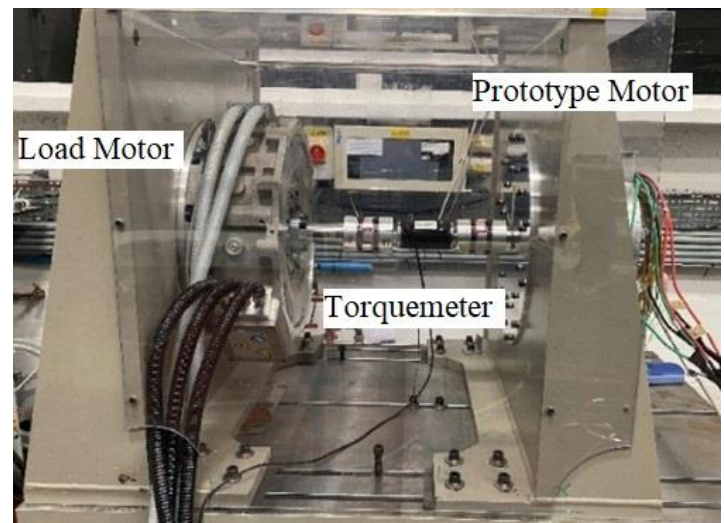

Fig. 16. Test rig: load motor (left hand side), SynRel prototype (right hand side).

\section{CONCLUSION}

In this paper a new geometrical parametrization for designing flux barriers of SynRel and PMaSynRel machines is presented. The proposed parametrization, based on spline curve, is explained in detail and optimizations are performed for both SynRel and PMaSynRel rotors.

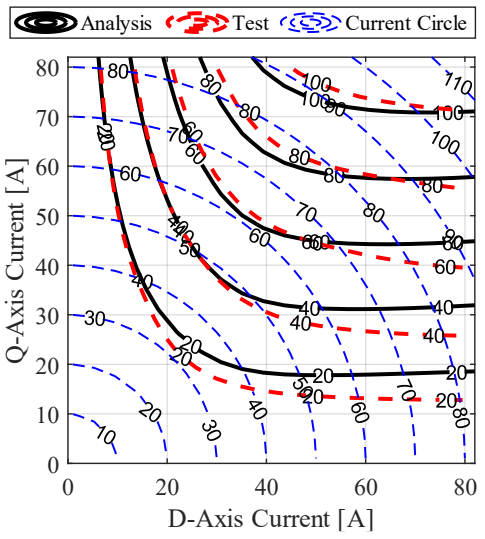

(a)

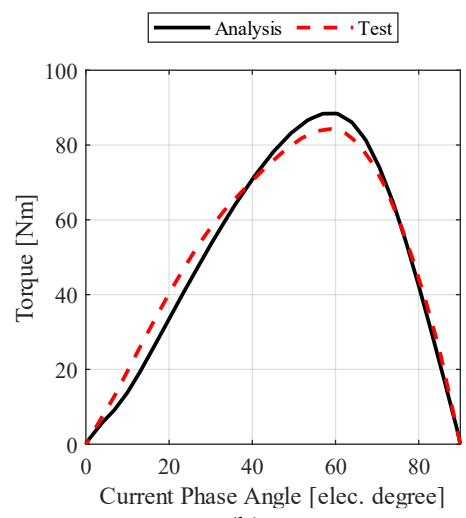

(b)

Fig. 17. Analysis and test results of the prototype machine: (a) torque isolines in DQ current plane, (b) current phase angle versus torque.

For the SynRel case, slightly better average torque is obtained with spline shaped barriers with a drawback of slightly higher torque ripple when compared to the state of art fluid shaped barriers. From mechanical point of view, fluid shaped barriers are superior to spline shaped barriers only because of the adopted rounded end-barrier shapes.

For the PMaSynRel case, using spline flux guides provide better average torque and slightly better torque ripple.

The comparative design optimization exercise reveals the potential of adopting a more complex flux barrier parametrization. Indeed, comparing the optimal geometries of the proposed flux barrier with the state of art solution, the former allows further decreasing the q-axis inductance without compromising the d-axis. This incremental improvement is due to the additional degrees of freedom of the spline flux barrier leading to a wider thickness of the innermost barrier. Clearly, the obtained improvement comes at the cost of a considerable higher computational burden of the design optimization.

A prototype of the optimal SynRel machine has been built and tested with different current modules and phase angles. The comparison between the test and FEA results reveals an acceptable matching of the performance characteristics. 


\section{REFERENCES}

[1] J. K. Kostko, "Polyphase reaction synchronous motors," in Journal of the American Institute of Electrical Engineers, vol. 42, no. 11, pp. 1162-1168, Nov. 1923, doi: 10.1109/JoAIEE.1923.6591529.J. Clerk Maxwell, A Treatise on Electricity and Magnetism, 3rd ed., vol. 2. Oxford: Clarendon, 1892, pp.68-73.

[2] A. Fratta, A. Vagati and F. Villata, "On the evolution of AC machines for spindle drive applications," Conference Record of the IEEE Industry Applications Society Annual Meeting,, San Diego, CA, USA, 1989, pp. 699704 vol.1, doi: 10.1109/IAS.1989.96725.

[3] D. Platt, "Reluctance motor with strong rotor anisotropy," Conference Record of the 1990 IEEE Industry Applications Society Annual Meeting, Seattle, WA, USA, 1990, pp. 224-229 vol.1, doi: 10.1109/IAS.1990.152190.

[4] D. A. Staton, T. J. E. Miller and S. E. Wood, "Maximising the saliency ratio of the synchronous reluctance motor," in IEE Proceedings B - Electric Power Applications, vol. 140, no. 4, pp. 249-259, July 1993, doi: 10.1049/ipb.1993.0031

[5] T. Matsuo and T. A. Lipo, "Rotor design optimization of synchronous reluctance machine," in IEEE Transactions on Energy Conversion, vol. 9, no. 2, pp. 359-365, June 1994, doi: 10.1109/60.300136.

[6] A. Tessarolo, "Modeling and Analysis of Synchronous Reluctance Machines With Circular Flux Barriers Through Conformal Mapping," in IEEE Transactions on Magnetics, vol. 51, no. 4, pp. 1-11, April 2015, Art no. 8104411, doi: 10.1109/TMAG.2014.2363434.

[7] F. Cupertino, G. Pellegrino and C. Gerada, "Design of Synchronous Reluctance Motors With Multiobjective Optimization Algorithms," in IEEE Transactions on Industry Applications, vol. 50, no. 6, pp. 3617-3627, Nov.Dec. 2014, doi: 10.1109/TIA.2014.2312540.

[8] G. Pellegrino, F. Cupertino and C. Gerada, "Barriers shapes and minimum set of rotor parameters in the automated design of Synchronous Reluctance machines," 2013 International Electric Machines \& Drives Conference, Chicago, IL, 2013, pp. 1204-1210, doi: 10.1109/IEMDC.2013.6556286.

[9] G. Pellegrino, F. Cupertino and C. Gerada, "Automatic Design of Synchronous Reluctance Motors Focusing on Barrier Shape Optimization," in IEEE Transactions on Industry Applications, vol. 51, no. 2, pp. 1465-1474, March-April 2015, doi: 10.1109/TIA.2014.2345953.

[10] R. Moghaddam and F. Gyllensten, "Novel High-Performance SynRM Design Method: An Easy Approach for A Complicated Rotor Topology," in IEEE Transactions on Industrial Electronics, vol. 61, no. 9, pp. 5058-5065, Sept. 2014, doi: 10.1109/TIE.2013.2271601.

[11] R. Rajabi Moghaddam, 'Synchronous Reluctance Machine (SynRM) in Variable Speed Drives (VSD) Applications', PhD dissertation, Stockholm, 2011.

[12] M. Gamba, G. Pellegrino and F. Cupertino, "Optimal number of rotor parameters for the automatic design of Synchronous Reluctance machines," 2014 International Conference on Electrical Machines (ICEM), Berlin, 2014, pp. 1334-1340, doi: 10.1109/ICELMACH.2014.6960355.

[13] E. Howard, M. J. Kamper and S. Gerber, "Asymmetric Flux Barrier and Skew Design Optimization of Reluctance Synchronous Machines," in IEEE Transactions on Industry Applications, vol. 51, no. 5, pp. 3751-3760, Sept.Oct. 2015, doi: 10.1109/TIA.2015.2429649.

[14] M. S. Mirazimi and A. Kiyoumarsi, "Magnetic Field Analysis of SynRel and PMASynRel Machines With Hyperbolic Flux Barriers Using Conformal Mapping," in IEEE Transactions on Transportation Electrification, vol. 6, no. 1, pp. 52-61, March 2020, doi: 10.1109/TTE.2019.2959400.

[15] R. M. R. Kumar et al., "Enhancing the Torque Density of Conventional PMSynRel Machine with Hybrid Flux Barrier," IECON 2019 - 45th Annual Conference of the IEEE Industrial Electronics Society, Lisbon, Portugal, 2019, pp. 4347-4352, doi: 10.1109/IECON.2019.8926624.

[16] P. Alotto, M. Barcaro, N. Bianchi and M. Guarnieri, "Optimization of Interior PM Motors With Machaon Rotor Flux Barriers," in IEEE Transactions on Magnetics, vol. 47, no. 5, pp. 958-961, May 2011, doi: 0.1109/TMAG.2010.2073450

[17] N. Bianchi, M. Degano and E. Fornasiero, "Sensitivity Analysis of Torque Ripple Reduction of Synchronous Reluctance and Interior PM Motors," in IEEE Transactions on Industry Applications, vol. 51, no. 1, pp. 187-195, Jan.Feb. 2015, doi: 10.1109/TIA.2014.2327143.

[18] C. Liu, K. Wang, S. Wang, Y. Wang and J. Zhu, "Torque ripple reduction of synchronous reluctance machine by using asymmetrical barriers and hybrid magnetic core," in CES Transactions on Electrical Machines and Systems, vol. 5, no. 1, pp. 13-20, March 2021, doi: 10.30941/CESTEMS.2021.00003.

[19] M. Murataliyev, M. Degano and M. Galea, "A Novel Sizing Approach for Synchronous Reluctance Machines," in IEEE Transactions on Industrial Electronics, vol. 68, no. 3, pp. 2083-2095, March 2021, doi: 10.1109/TIE.2020.2975461.

[20] S. Ferrari and G. Pellegrino, "FEAfix: FEA Refinement of Design Equations for Synchronous Reluctance Machines," in IEEE Transactions on Industry Applications, vol. 56, no. 1, pp. 256-266, Jan.-Feb. 2020, doi: 10.1109/TIA.2019.2954797.

[21] G. Gallicchio et al., "High Speed Synchronous Reluctance Machines: Modeling, Design and Limits," in IEEE Transactions on Energy Conversion, doi: 10.1109/TEC.2021.3086879.

[22] A. Vagati, M. Pastorelli, G. Francheschini and S. C. Petrache, "Design of lowtorque-ripple synchronous reluctance motors," in IEEE Transactions on Industry Applications, vol. 34, no. 4, pp. 758-765, July-Aug. 1998, doi: $10.1109 / 28.703969$

[23] A. Vagati, G. Franceschini, I. Marongiu, G. P. Troglia, "Design criteria of high performance synchronous reluctance motors", Industry Applications Society Annual Meeting, Conference Record of the IEEE, 4 - 9 Oct., 1992, Page(s): 66 - 73 vol.1.

[24] G. Poloni and V. Pediroda, "GA Coupled with Computationally Expensive Simulations: Tools to Improve Efficiency" in Genetic Algorithms and Evolution Strategy in Engineering and Computer Science: Recent Advances and Industrial Applications, D. Quagliarella, J. Periaux, C. Poloni, G. Winter, John Wiley\&Sons, 2000.

[25] I.M.Sobol, "Distribution of points in a cube and approximate evaluation of integrals", U.S.S.R Comput.Maths.Math.Phys 7:86-112

[26] M. Di Nardo et al., "End barrier shape optimizations and sensitivity analysis of synchrnous reluctance machines," IECON 2015 - 41st Annual Conference of the IEEE Industrial Electronics Society, Yokohama, Japan, 2015, pp. 002914-002919, doi: 10.1109/IECON.2015.7392545.

[27] M. Barcaro, G. Meneghetti, N. Bianchi, "Structural Analysis of the Interior PM Rotor Considering Both Static and Fatigue Loading ", in IEEE Transactions on Industry Applications, Vol. 50, No. 1 , 2014, pp. 253 - 260.

[28] E. Armando, R. I. Bojoi, P. Guglielmi, G. Pellegrino and M. Pastorelli, "Experimental Identification of the Magnetic Model of Synchronous Machines," in IEEE Transactions on Industry Applications, vol. 49, no. 5, pp. 2116-2125, Sept.-Oct. 2013, doi: 10.1109/TIA.2013.2258876.

\section{BIOGRAPHIES}

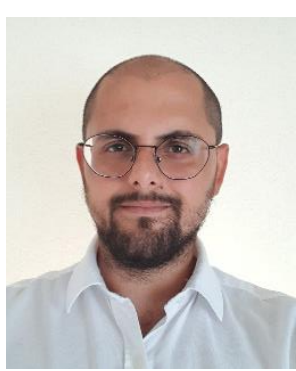

Oğuz Korman received the B.Sc. and M.Sc. in Electrical Engineering from Istanbul Technical University, Turkey in 2015 and 2018, respectively. Between 2017-2019 he worked as an electromagnetic design engineer in Istanbul, Turkey involving in many projects focusing on industrial and custom applications. He is currently studying towards Ph.D. degree with Power Electronics, Machines and Control (PEMC) Group, University of Nottingham, UK. His research interests are modelling and design of highperformance electric motors for industrial and traction applications.

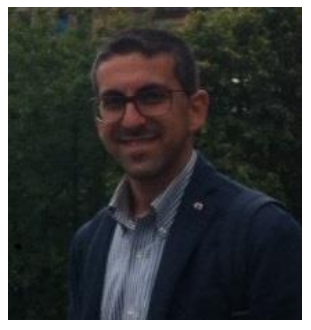

Mauro Di Nardo (Member, IEEE) received the M.Sc. (Hons.) degree in electrical engineering from the Polytechnic University of Bari, Italy, in 2012, and the Ph.D. degree in electrical machine design from the University of Nottingham, U.K., in 2017. From 2017 to 2019, he was Head with the AROL R\&D Team within the Polytechnic University of Bari leading industrial projects on electrical drives design for mechatronics applications. Since the 2019, he is with the Power Electronics and Machine Control Group of the University of Nottingham as Research Fellow working on wide variety of projects of high industrial and scientific impacts. His research interests include the analysis, modelling, and design optimizations of permanent magnet and synchronous reluctance 
machines for automotive, aerospace and household sectors, induction motor for industrial applications as well as niche machine topologies such as bearingless and hysteresis motor.

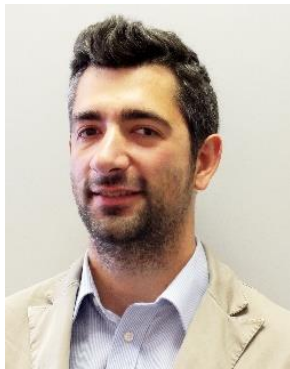

Michele Degano (M'15) received his Master's degree in Electrical Engineering from the University of Trieste, Italy, in 2011, and his Ph.D. degree in Industrial Engineering from the University of Padova, Italy, in 2015. Between 2014 and 2016, he was a postdoctoral researcher at The University of Nottingham, UK, where he joined the Power Electronics, Machines and Control (PEMC) Research Group. In 2016 he was appointed Assistant Professor in Advanced Electrical Machines, at The University of Nottingham, UK. He was promoted Associate Professor in 2020. Dr. Degano is currently the PEMC Director of Industrial Liaison leading research projects for the development of future hybrid electric aerospace platforms and electric transports. He is author/co-author of more than 100 scientific papers and an Associate Editor of the IEEE Transactions on Energy Conversion. His main research focuses on electrical machines and drives for industrial, automotive, railway and aerospace applications, ranging from small to large power.

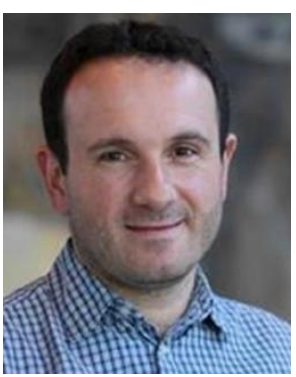

Chris Gerada (SM'12) is an Associate Pro-Vice Chancellor for Industrial Strategy and Impact and Professor of Electrical Machines. His principal research interest lies in electromagnetic energy conversion in electrical machines and drives, focusing mainly on transport electrification. He has secured over $£ 20 \mathrm{M}$ of funding through major industrial, European and UK grants and authored more than 350 referred publications. He received the $\mathrm{Ph} . \mathrm{D}$. degree in numerical modelling of electrical machines from The University of Nottingham, Nottingham, U.K., in 2005. He subsequently worked as a Researcher with The University of Nottingham on high-performance electrical drives and on the design and modelling of electromagnetic actuators for aerospace applications. In 2008, he was appointed as a Lecturer in electrical machines; in 2011, as an Associate Professor; and in 2013, as a Professor at The University of Nottingham. He was awarded a Research Chair from the Royal Academy of Engineering in 2013. Prof. Gerada served as an Associate Editor for the IEEE TRANSACTIONS ON INDUSTRY APPLICATIONS and is the past Chair of the IEEE IES Electrical Machines Committee 